\title{
Irradiation des cristallins des patients par scanners de perfusion itératifs : dosimétrie et optimisation
}

\author{
J. Guersen ${ }^{1 \text { a }}$, L. Cassagnes ${ }^{1,2}$, G. Méchin ${ }^{1}$, C. Etard $^{3}$, J.-L. Réhel ${ }^{3}$, B. Jean ${ }^{1}$, E. Chabert ${ }^{1}$, J. Gabrillargues ${ }^{1,2}$, \\ M. Labattu ${ }^{1}$, L. Boyer ${ }^{1,2}$ et P. Chabrot ${ }^{1,2}$ \\ 1 Pôle de Radiologie et Imagerie Médicale, CHU Clermont-Ferrand, 63000 Clermont-Ferrand, France. \\ 2 ISIT, UMR 6284 UdA-CNRS, 63001 Clermont-Ferrand, France. \\ 3 IRSN, PRP-HOM/SER/UEM, 92262 Fontenay-aux-Roses, France.
}

Reçu le 26 août 2013 - Accepté le 5 février 2014

\begin{abstract}
Résumé - Le cristallin est un organe radiosensible. En avril 2011, la Commission Internationale de Protection Radiologique (CIPR) fixait à 500 mGy la nouvelle dose seuil d'apparition d'effets déterministes au cristallin. Les patients qui présentent un anévrysme intracérébral rompu bénéficient d'examens scanographiques de l'encéphale (CT) justifiés, pour le diagnostic et le suivi post traitement, réalisé le plus souvent par embolisation radioguidée. Ces examens itératifs exposent le cristallin aux rayons X. Cet article chiffre l'exposition des cristallins de 18 patients ayant bénéficié en 2011 d'examens scanographiques encéphaliques suite à une rupture d'anévrysme intracérébral, et propose un moyen de diminuer cette exposition. Dans notre étude, $44 \%$ des patients étudiés ont reçu une dose supérieure à $500 \mathrm{mGy}$ au niveau des yeux, générée par les examens scanographiques et en particulier les CT de perfusion, indispensables pour évaluer le risque d'ischémie retardée par vasospasme. Nous avons observé qu'en positionnant les patients en hyper flexion cervicale lors de la réalisation des scanners de perfusion, la dose aux cristallins pouvait être diminuée de près de $50 \%$.
\end{abstract}

\begin{abstract}
Eye lens irradiation by CT perfusion: dosimetry and optimization. The lens of the eye is a radiosensitive organ. In April 2011, the International Commission on Radiological Protection (ICRP) set the new threshold dose of occurrence of deterministic lens effects to $500 \mathrm{mGy}$. Patients with a ruptured intracranial aneurysm are imaged by CT scans of the brain (CT), for diagnosis and post-treatment follow-up; embolization is the standard treatment. These iterative examinations expose the lens to X-rays. This paper evaluates the lens X-ray exposure of 18 patients who underwent encephalic CT scans after a ruptured intracranial aneurysm in 2011, and proposes a method to reduce this exposure. In our study, $44 \%$ of patients received eye doses greater than $500 \mathrm{mGy}$, generated by CT examinations, especially cerebral CT perfusion, necessary to assess the risk of delayed vasospasm/ischemia. By positioning the patient in neck hyperflexion for cerebral CT perfusion, the dose could be reduced by around $50 \%$.
\end{abstract}

Keywords: eye lens dose / CT perfusion / TLD thermoluminescent dosimeter / optimization

\section{Introduction}

Le cristallin est un organe radiosensible. En 2007, la publication numérotée 103 de la Commission Internationale de Protection Radiologique (ICRP, 2007) précisait que le seuil de dose reçue lors d'une exposition brève et unique pour l'apparition d'opacités détectables au cristallin était compris entre 0,5 et $2 \mathrm{~Gy}$. La dose seuil pour la déficience visuelle (cataracte) était de 5 Gy. En avril 2011, le Statement on Tissue Reactions, approuvé par la CIPR le 21 avril 2011 (ICRP, 2011), précisait que "for the lens of the eye, the threshold in absorbed dose is now considered to be 0,5 Gy».

Cet abaissement de la dose seuil de risque d'apparition de la cataracte radio-induite nous a conduit à nous questionner

\footnotetext{
a jguersen@chu-clermontferrand.fr
}

sur la dose absorbée que recevaient les cristallins des patients qui bénéficiaient d'embolisations d'anévrysmes intracrâniens rompus, dont le diagnostic et la surveillance post interventionnelle étaient assurés par des scanners standard et/ou de perfusion itératifs (Michel et al., 2012).

En effet, le protocole de prise en charge de ces patients, dont le pronostic est grevé par un risque de 30 à $60 \%$ (King, 1997) d'ischémie retardée par vasospasme (50\% des patients ayant souffert d'une ischémie retardée conserveront un handicap définitif), prévoit après traitement la réalisation de scanners de perfusion pour évaluer le retentissement d'aval dès le début des signes cliniques et adapter la prise en charge (Vajkoczy et al., 2003). Le pronostic vital et fonctionnel d'un malade après une rupture d'anévrysme est lié à la gravité initiale de l'hémorragie, mais aussi secondairement à la sévérité 
de ce vasospasme. Plusieurs études (Dankbaar et al., 2009; Greenberg et al., 2010; Kistka et al., 2013) ont montré que le scanner de perfusion était un outil sensible et spécifique dans la détection précoce et le suivi du vasospasme, et les auteurs concluent en soulignant ses grande disponibilité et rapidité de mise en œuvre pour les patients de réanimation.

C'est pourquoi dans notre institution nous réalisons un scanner de perfusion chez ces patients à haut risque, chaque fois que des signes de vasospasme sont détectés.

$\mathrm{Au}$ cours du $1^{\mathrm{er}}$ semestre 2011 dans notre établissement, sur un échantillon de 10 patients embolisés en neuroradiologie interventionnelle, nous avons constaté que la moyenne d'examens scanographiques (CT) réalisés entre $\mathrm{J}-1$ et $\mathrm{J}+30$ après l'embolisation était de 2,3 CT de perfusion + 1,7 CT standard, nous amenant à nous interroger sur la dose cumulée sur un mois par ces procédures (embolisation et scanners) : étaitelle supérieure au seuil de $500 \mathrm{mGy}$, et si oui, existait-il des moyens de la réduire?

\section{Matériel et méthode}

L'étude a porté, entre le 24 juin 2011 et le 22 septembre 2011, sur 18 patients ayant bénéficié d'une embolisation par voie endovasculaire d'un anévrysme intracérébral rompu. En moyenne, 1,5 CT standard et 1,6 CT de perfusion ont été réalisés chez ces 18 patients entre le jour précédent l'embolisation et le mois qui suivait.

\subsection{Exposition aux rayons $X$ des cristallins des patients dans le cadre de leur prise en charge médicale}

L'embolisation par voie endovasculaire des anévrysmes intracérébraux est réalisée sous guidage radioscopique, sur une installation de radiologie interventionnelle biplan GE Healthcare Advant'x (1999) équipée de 2 amplificateurs de brillance.

Par ailleurs, pour le diagnostic ou la surveillance post interventionnelle, les patients bénéficient d'examens scanographiques (CT) de l'encéphale, effectués sur un scanner GE Healthcare Discovery 750 HD $^{\circledR}$ (2009) équipé de 64 barrettes et muni d'un système de reconstruction itératif. Deux types d'examens CT sont réalisés : des examens CT standard et/ou des examens CT de perfusion.

- Un examen CT standard est une exploration scanographique simple de l'encéphale réalisée en mode hélicoïdal avec une seule acquisition. Les CTDI vol (Indice de Dose Scanographique Volumique) et PDL (Produit Dose Longueur) d'un examen CT standard du crâne fixés par l'arrêté du 24 octobre 2011 relatif aux niveaux de référence diagnostiques en radiologie et en médecine nucléaire ${ }^{1}$ sont 65 mGy et 1050 mGy.cm.

- Un examen CT de perfusion (pour lequel il n'existe pas de niveaux de référence diagnostiques) est une exploration plus complexe de l'encéphale qui combine 3 phases :

- un CT standard sans injection de produit de contraste;

\footnotetext{
1 NOR : ETSP1129093A.
}

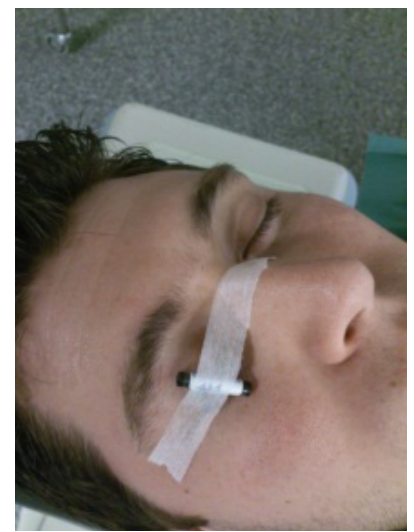

Fig. 1. Dosimètre TLD sur l'œil droit pendant une embolisation dans la salle d'angiographie.

TLD dosimeter over the right eye during embolization.

- une exploration dite de perfusion réalisée avec injection de produit de contraste et acquisitions hélicoïdales itératives en mode navette (sur notre installation, le CTDI vol de l'exploration dite de perfusion était de $274 \mathrm{mGy}$ );

- un passage hélicoïdal ciblé sur le polygone de Willis associé à un CTDI vol de 64 mGy.

La dose totale délivrée aux cristallins est donc la conséquence d'une part de l'embolisation, et d'autre part des examens CT itératifs de l'encéphale.

\subsection{Mesure de la dose aux cristallins lors des embolisations intracrâniennes endovasculaires}

La dose délivrée aux cristallins lors de l'embolisation a été mesurée pour les 18 patients à l'aide de dosimètres thermoluminescents (TLD) de poudre de FLi:Mg,Cu,P type GR207P. (Dong et al., 2002). Un tube de poudre de FLi était positionné sur l'œil droit de chaque patient, le plus proche du tube à rayons $\mathrm{X}$ latéral, situé à droite du patient sur l'installation radiologique du service (Fig. 1).

L'étalonnage et la lecture des dosimètres TLD ont été effectués par l'IRSN. L'étalonnage a été réalisé selon la norme ISO-4037 parties 1 et 2, pour le faisceau W80, d'énergie moyenne $57 \mathrm{keV}$, proche de celle des faisceaux utilisés en neuroradiologie interventionnelle. Les résultats fournis par l'IRSN étaient exprimés en équivalent de dose sous $0,07 \mathrm{~mm}$ de tissus $\mathrm{H}_{\mathrm{p}}(0,07)$. L'incertitude sur la mesure est estimée par le laboratoire d'étalonnage à $10 \%(1 \sigma)$. Nous avons considéré qu'en $1^{\text {ère }}$ approximation, ils permettaient d'estimer la dose au cristallin.

Bien que la lecture des dosimètres TLD se fasse en différé, ces dosimètres ont été choisis pour leur facilité d'utilisation pour des mesures sur patient en neuroradiologie interventionnelle (pas d'alimentation ni de câble électrique).

\subsection{Mesure de la dose aux cristallins lors des examens scanographiques de l'encéphale}

L'estimation de la dose aux cristallins lors des examens CT pour chacun des 18 patients a été réalisée à partir de mesures 


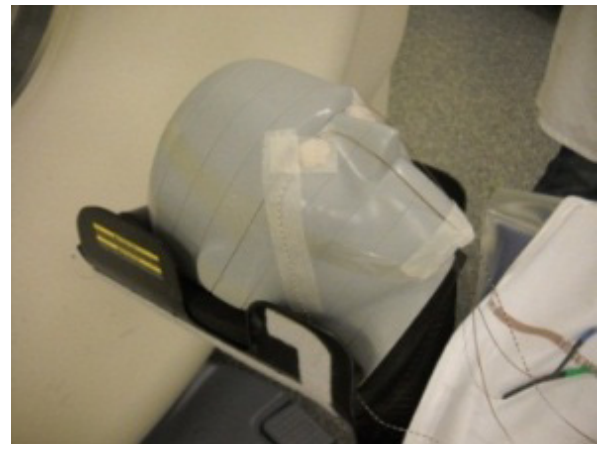

Fig. 2. MOSFETs sur le fantôme crânien pendant un examen CT. MOSFETs on the head phantom during a CT examination.

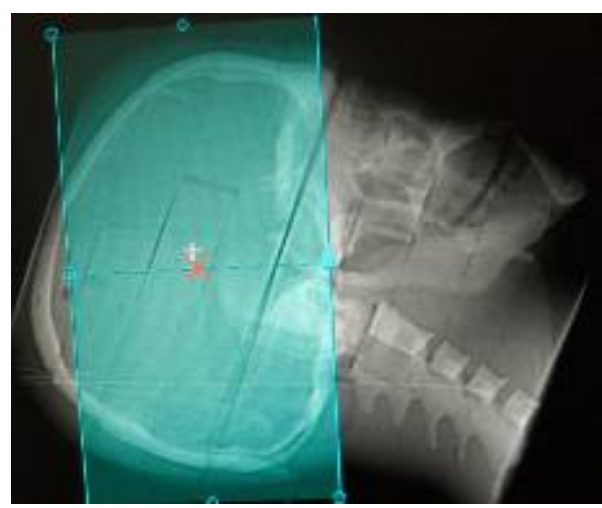

Fig. 3. Positionnement des coupes de scanner sur le fantôme crânien : tête en hyperflexion.

Positioning of scanner cuts on the head phantom: head in hyperflexion.

sur un fantôme crânien anthropomorphe $\mathrm{CIRS}^{\circledR}$, à l'aide d'un système de dosimétrie mobile MOSFET ${ }^{\circledR}$ (Metal Oxide Semiconductor Field Effect Transistor Best Medical Canada) muni de détecteurs TN-502 RDM de type transistor à effet de champ (5 au maximum). Un détecteur TN-502 RDM était positionné sur chaque œil, sous $3 \mathrm{~mm}$ de cire, pour estimer au mieux la dose effectivement reçue par les cristallins (Fig. 2).

Les détecteurs TN-502RDM ont été étalonnés individuellement le 11 janvier 2011 selon la norme ISO-4037 parties 1 et 2, pour les faisceaux W80 et W110, en kerma dans l'air et en équivalent de dose $\mathrm{H}_{\mathrm{p}}(0,07)$, d'énergies moyennes respectives 57 et $79 \mathrm{keV}$, proches de celles des faisceaux utilisés en scanographie.

Dans un premier temps, les mesures de dose au cristallin ont été réalisées dans les conditions techniques habituelles, avec les paramètres d'acquisition usuels. Dans ce cas, les yeux du fantôme se trouvaient dans le faisceau direct de rayons $\mathrm{X}$ pour toutes les phases des examens.

Dans un second temps, d'autres conditions de réalisation ont été évaluées afin de réduire la dose aux cristallins :

- la tête du fantôme a été positionnée en hyper flexion cervicale (menton proche de la poitrine) pour «sortir» les yeux du champ d'exploration pendant la phase de l'examen dite « de perfusion » (Fig. 3). Tous les autres paramètres d'acquisition des images étaient par ailleurs ceux des conditions cliniques habituelles.

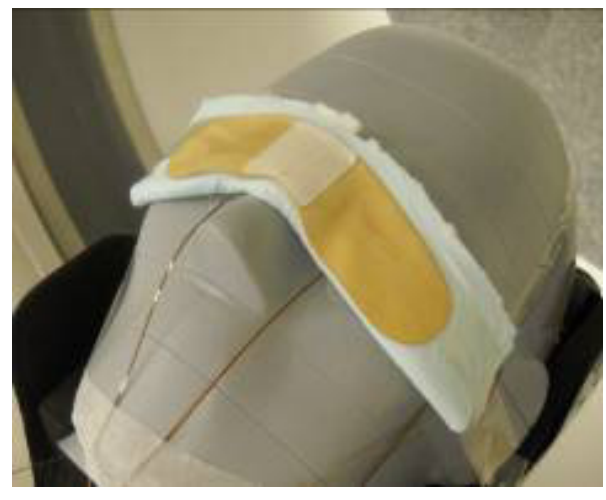

Fig. 4. Protections oculaires au bismuth sur le fantôme. Bismuth eye shield on the head phantom.

- Des protections oculaires au bismuth ont ensuite été positionnées, en maintenant la tête en hyper flexion (Fig. 4). Ces protections ont été placées sur les yeux du fantôme après la réalisation des topogrammes (le topogramme ou scout-view est une image radiographique numérisée obtenue par déplacement du patient devant les détecteurs du scanner en position fixe, et qui sert à placer les coupes en fonction de l'organe ou de la pathologie à explorer). Elles n'ont été utilisées que lors de la phase «de perfusion » pendant laquelle les yeux n'étaient pas dans le faisceau direct. En effet, l'équipe médicale souhaitait éviter tout artéfact dans l'image lié à la présence des caches oculaires au bismuth dans le champ exploré.

Le système mobile MOSFET ${ }^{\circledR}$ a été choisi pour cette partie de l'étude afin de disposer de résultats en temps réel, permettant d'étudier l'impact dosimétrique de ces différentes conditions de réalisation scanographiques.

Toutes les acquisitions scanographiques ont été doublées afin d'évaluer la reproductibilité des mesures. L'incertitude sur les mesures effectuées avec les détecteurs MOSFET ${ }^{\circledR}$ est estimée à $25 \%(1 \sigma)$ compte tenu de l'importante variation de leur réponse dans la gamme d'énergie utilisée (Bassinet et al., 2013).

\section{Résultats}

\subsection{Estimation des doses délivrées au cristallin}

Pour les 18 patients étudiés, la dose équivalente au cristallin droit générée par les embolisations endovasculaires était en moyenne de $147 \mathrm{mSv}$ (min. $11 \mathrm{mSv}$ et max. $1293 \mathrm{mSv}$ ). Le détail des mesures, présenté dans le tableau 1, montre une grande variabilité des doses équivalentes au cristallin, liée d'une part à la complexité de l'intervention pouvant nécessiter des temps de radioscopie et des nombres d'acquisitions radiographiques très variables d'un patient à l'autre, et d'autre part à la localisation anatomique de l'anévrysme par rapport aux yeux.

Un examen CT standard de l'encéphale, sans injection de produit de contraste, délivrait aux cristallins $47+/-12$ mGy (pas d'écart significatif entre cristallins droit et gauche) pour une haute tension de $120 \mathrm{kV}$ et un index de dose scanographique en volume $\left(\mathrm{CTDI}_{\mathrm{vol}}\right)$ égal à $34 \mathrm{mGy}$. Les paramètres dosimétriques des scanners cérébraux standard $\left(\mathrm{CTDI}_{\mathrm{vol}}\right.$ et 
Tableau 1. Doses aux cristallins générées par les embolisations et les examens CT standard et de perfusion entre $\mathrm{J}-1$ et $\mathrm{J}+30$ après l'embolisation.

Doses to eye lenses generated by embolization and standard and perfusion CT examinations between $\mathrm{J}-1$ and $\mathrm{J}+30$ after embolization.

\begin{tabular}{|c|c|c|c|c|c|}
\hline & \multicolumn{2}{|c|}{ Embolisation } & \multicolumn{3}{|c|}{ Examens scanographiques } \\
\hline & Date $(\mathbf{J})$ & $\begin{array}{l}\text { Dose équivalente } \\
\text { au cristallin droit } \\
(\mathrm{mSv})\end{array}$ & $\begin{array}{c}\text { Dose liée aux CT } \\
\text { standards (mGy) } \\
\text { [nombre d'examens] }\end{array}$ & $\begin{array}{c}\text { Dose liée aux CT } \\
\text { de perfusion (mGy) } \\
\text { [nombre d'examens] }\end{array}$ & $\begin{array}{r}\text { Dose totale } \\
\text { liée aux CT } \\
\text { (mGy) }\end{array}$ \\
\hline Patient $n^{\circ} 1$ & $24 / 06 / 2011$ & 254 & $235[5]$ & $620[2]$ & 855 \\
\hline Patient $n^{\circ} 2$ & $05 / 07 / 2011$ & 51 & $47[1]$ & $310[1]$ & 357 \\
\hline Patient $n^{\circ} 3$ & 07/07/2011 & 65 & $47[1]$ & $620[2]$ & 667 \\
\hline Patient $n^{\circ} 4$ & $12 / 07 / 2011$ & 19 & 0 & 0 & 0 \\
\hline Patient $n^{\circ} 5$ & $12 / 07 / 2011$ & 25 & 0 & 0 & 0 \\
\hline Patient $n^{\circ} 6$ & $19 / 07 / 2011$ & 43 & $47[1]$ & $930[3]$ & 977 \\
\hline Patient $\mathrm{n}^{\circ} 7$ & $29 / 07 / 2011$ & 71 & $282[6]$ & $1240[6]$ & 1522 \\
\hline Patient $n^{\circ} 8$ & $25 / 07 / 2011$ & 48 & $141[3]$ & 310 [3] & 451 \\
\hline Patient $n^{\circ} 9$ & 04/08/2011 & 47 & 188 [4] & 1240 [4] & 1428 \\
\hline Patient $\mathrm{n}^{\circ} 10$ & $09 / 08 / 2011$ & 92 & 0 & 0 & 0 \\
\hline Patient $\mathrm{n}^{\circ} 11$ & $11 / 08 / 2011$ & 46 & 0 & 0 & 0 \\
\hline Patient $n^{\circ} 12$ & $12 / 08 / 2011$ & 82 & 0 & 1550 [5] & 1550 \\
\hline Patient $n^{\circ} 13$ & $23 / 08 / 2011$ & 67 & 94 [2] & 0 & 94 \\
\hline Patient $\mathrm{n}^{\circ} 14$ & $27 / 08 / 2011$ & 121 & 94 [2] & 1550 [5] & 1644 \\
\hline Patient $n^{\circ} 15$ & $13 / 09 / 2011$ & 11 & $47[1]$ & 0 & 47 \\
\hline Patient $\mathrm{n}^{\circ} 16$ & $20 / 09 / 2011$ & 46 & 0 & 0 & 0 \\
\hline Patient $\mathrm{n}^{\circ} 17$ & $20 / 09 / 2011$ & 1293 & $47[1]$ & 620 [2] & 667 \\
\hline Patient $\mathrm{n}^{\circ} 18$ & $22 / 09 / 2011$ & 257 & 0 & 0 & 0 \\
\hline
\end{tabular}

Tableau 2. Doses aux cristallins délivrées lors d'un examen CT de perfusion, réalisées dans les conditions cliniques habituelles (yeux dans la zone directement exposée pour les 3 phases de l'examen).

Doses to eye lenses delivered during a CT perfusion examination, in the usual clinical conditions (eyes in the area directly exposed to the 3 phases of the exam).

\begin{tabular}{cccc}
\hline $\begin{array}{c}\text { Phase de } \\
\text { l'examen CT de } \\
\text { perfusion }\end{array}$ & $\begin{array}{c}\text { Haute } \\
\text { tension } \\
(\mathbf{k V})\end{array}$ & $\begin{array}{c}\text { Index de dose } \\
\mathbf{C T D I}_{\text {vol }} \\
(\mathbf{m G y}\end{array}$ & $\begin{array}{c}\text { Dose aux } \\
\text { cristallins } \\
(\mathbf{m G y})[\mathbf{1} \boldsymbol{\sigma}]\end{array}$ \\
\hline Crâne standard sans IV & 120 & 34 & $47[12]$ \\
«Perfusion » & 80 & 274 & $170[43]$ \\
\hline «Polygone de Willis » & 140 & 64 & $91[23]$ \\
TOTAL & & & $\mathbf{3 0 8}[\mathbf{5 0}]$ \\
\hline
\end{tabular}

DLP - Dose Length Product) étaient en deçà des niveaux de référence diagnostiques fixées par la réglementation française ${ }^{1}$.

La dose aux cristallins (droit et gauche) due à un examen CT de perfusion réalisé dans les conditions techniques habituelles a été estimée à $310+/-50$ mGy. Le tableau 2 présente les doses aux cristallins liées aux différentes phases de l'examen. Les paramètres d'acquisition utilisés pour les différentes phases figurent également dans le tableau 2. Il n'existe pas de Niveaux de Référence Diagnostiques (NRD) réglementaires pour les CT de perfusion.

Pour les 18 patients étudiés, le tableau 1 présente la somme des doses délivrées aux cristallins par l'ensemble des examens CT (standard et de perfusion) effectués entre $\mathrm{J}-1$ et $\mathrm{J}+30$ (J : jour de l'embolisation). La dose totale moyenne s'élevait à 570 mGy (min. 0 mGy et max. 1644 mGy). Parmi les 12 patients ayant bénéficié d'au moins un examen CT, 8 ont reçu une dose aux cristallins supérieure à 500 mGy (67\%).

De plus, on note que les doses délivrées par les examens CT sous estiment nécessairement la dose réellement reçue par
Tableau 3. Doses aux cristallins délivrées lors d'un examen CT de perfusion (tête en hyper flexion et cristallins hors du faisceau direct lors de la phase de perfusion).

Doses to eye lenses issued during a perfusion CT examination (head in hyperflexion and eye lenses outside the direct beam during the perfusion phase).

\begin{tabular}{cccc}
\hline $\begin{array}{c}\text { Phase de } \\
\text { l'examen CT de } \\
\text { perfusion }\end{array}$ & $\begin{array}{c}\text { Haute } \\
\text { tension } \\
(\mathbf{k V})\end{array}$ & $\begin{array}{c}\text { Index de dose } \\
\mathbf{C T D I}_{\text {vol }} \\
(\mathbf{m G y})\end{array}$ & $\begin{array}{c}\text { Dose aux } \\
\text { cristallins } \\
(\mathbf{m G y})[\mathbf{1} \boldsymbol{\sigma}]\end{array}$ \\
\hline Crâne standard sans IV & 120 & 32 & $41[11]$ \\
«Perfusion» & 80 & 274 & $40[10]$ \\
\hline «Polygone de Willis» & 140 & 64 & $83[21]$ \\
TOTAL & & & $\mathbf{1 6 5}[\mathbf{2 6}]$ \\
\hline
\end{tabular}

les cristallins et notamment le cristallin droit des patients, le plus exposé aux rayons X lors de l'embolisation.

\subsection{Impact dosimétrique des différentes conditions de réalisation des examens CT}

Les mesures ont montré que positionner la tête en hyper flexion permettait de réduire considérablement la contribution de la phase « de perfusion » à la dose aux cristallins : 40 mGy vs. $170 \mathrm{mGy}$.

Pour l'examen CT de perfusion (incluant les 3 phases), ces conditions de réalisation ont permis de réduire la dose totale de près de $50 \%$ (165 mGy vs. 310 mGy) comme l'indique le tableau 3 .

La mise en place de protections oculaires au bismuth, tout en maintenant la position du crâne en hyper flexion, a permis une réduction supplémentaire de la dose reçue par les cristallins durant la phase «de perfusion», de 40 à 26 mGy (Tab. 4). 
Tableau 4. Doses aux cristallins délivrées lors d'un examen CT de perfusion (tête en hyper flexion, cristallins hors du faisceau direct et protections oculaire au bismuth lors de la phase de perfusion).

Doses to eye lenses issued during a perfusion CT examination (head in hyperflexion, eye lenses outside the direct beam and bismuth eye shield during the perfusion phase).

\begin{tabular}{|cccc}
\hline $\begin{array}{c}\text { Phase de } \\
\text { l'examen CT de } \\
\text { perfusion }\end{array}$ & $\begin{array}{c}\text { Haute } \\
\text { tension } \\
(\mathbf{k V})\end{array}$ & $\begin{array}{c}\text { Index de dose } \\
\mathbf{C T D I}_{\text {vol }} \\
(\mathbf{m G y})\end{array}$ & $\begin{array}{c}\text { Dose aux } \\
\text { cristallins } \\
(\mathbf{m G y})[\mathbf{1} \boldsymbol{\sigma}]\end{array}$ \\
\hline Crâne standard sans IV & 120 & 34 & $47[12]$ \\
«Perfusion » & 80 & 274 & $26[7]$ \\
«Polygone de Willis » & 140 & 64 & $83[21]$ \\
TOTAL & & & $\mathbf{1 5 0}[\mathbf{2 5}]$ \\
\hline
\end{tabular}

Pour l'examen CT de perfusion, la dose aux cristallins était ainsi réduite de 165 à $150 \mathrm{mGy}$ (Tab. 4).

\section{Discussion}

La dose reçue par les cristallins de nos patients lors des examens CT de perfusion était significative, mais le bénéfice attendu de ces explorations pour les patients doit passer au premier plan. Ces examens étaient réalisés dans les conditions standards de routine. Positionner les patients en hyper flexion cervicale permet de réduire la dose de $50 \%$. La position en hyper flexion cervicale est compatible avec les recommandations du guide de la SFR (Société Française de Radiologie) «guide pratique à l'usage des médecins radiologues pour l'évaluation de leurs pratiques professionnelles » (SFR, 2009), qui stipule que la tête doit être fixée dans une position la plus proche possible de la ligne orbito-méatale. Une optimisation est toutefois possible si les yeux ne sont pas sont dans le champ d'acquisition (faisceau direct de rayons X). Ce positionnement est simple à mettre en œuvre, et ne perturbe pas la qualité des résultats attendus du scanner.

Le gain dosimétrique obtenu avec les protections au bismuth était faible (environ 15 mGy sur un total de 165). L'utilisation de ce dispositif induisait un risque d'artéfact dans l'image et de mouvement du patient au moment de la mise en place. Ces protections oculaires au bismuth ne nous sont pas apparues pertinentes. Nous avons choisi de ne pas les utiliser en routine et de ne pas les conseiller.

\section{Conclusion}

Notre étude a montré que les scanners standards et notamment les scanners de perfusion répétés, justifiés et prescrits pour la surveillance des embolisations d'anévrysmes intra crâniens rompus, étaient susceptibles d'exposer les cristallins des patients à des doses cumulées totales dépassant dans $44 \%$ des cas étudiés le nouveau seuil de 500 mGy fixé par la CIPR pour l'apparition d'effets déterministes radio induits aux cristallins. De plus, ce pourcentage n'incluait pas l'exposition de l'œil droit des patients générée par l'embolisation elle-même.
Notre étude a également mis en évidence la possibilité d'optimiser cette dose aux cristallins pendant les examens scanographiques en positionnant les patients en hyper flexion cervicale pendant la réalisation de la phase dite «de perfusion» des CT de perfusion. Ce positionnement permettait de «soustraire » les yeux du faisceau direct de rayons $\mathrm{X}$ et offrait ainsi la possibilité de réduire la dose reçue au niveau des cristallins de près de $50 \%$.

Le protocole de réalisation des scanners de perfusion de notre centre a donc été réécrit en insistant notamment sur l'hyper flexion de la tête des patients, afin que la limite inférieure de l'acquisition de la phase dite de perfusion se situe dans le plan méat-toit de l'orbite, et si possible $1 \mathrm{~cm}$ au-dessus pour éviter l'exposition directe des yeux et limiter l'effet de pénombre du faisceau.

\section{Références}

Bassinet C., Huet C., Baumann M., Etard C., Réhel J.L., Boisserie G., Debroas J., Aubert B., Clairand I. (2013) Characterization of MOSFET detectors for in vivo dosimetry in interventional radiology and for dose reconstruction in case of overexposure, Health Phys. 104 (4), 379-84.

Dankbaar J.W., Rijsdijk M., Van der Schaaf I.C., Velthuis B.K., Wermer M.J.H., Rinkel G.J.E. (2009) Relationship between vasospasm, cerebral perfusion, and delayed cerebral ischemia after aneurysmal subarachnoid hemorrhage, Neuroradiology 51, 813819.

Dong S.L., Chu T.C., Lan G.Y., Wu T.H., Lin Y.C., Lee J.S. (2002) Characterization of highsensitivity Metal Oxide Semiconductor Field Effect Transistor dosimeters system 12 and LiF:Mg,Cu,P Thermoluminescence dosimeters for use in diagnostic radiology, Appl. Radiat. Isotopes 57 (6), 883-891.

Greenberg E.D. et al. (2010) Diagnostic accuracy of CT angiography and CT perfusion for cerebral vasospasm: a meta-analysis, American Journal of Neuroradiology 31 (10), 1853-1860.

ICRP Publication 103 (2007) The 2007 Recommendations of the International Commission on Radiological Protection. Ann. ICRP 37.

ICRP (2011) Statement on Tissue Reactions. ICRP réf. 4825-30931464.

King J.T. (1997) Epidemiology of aneurysmal subarachnoid hemorrhage, Neuroimaging Clinics of North America 7 (4), 659-668.

Kistka H., Dewan M.C., Mocco J. (2013) Evidence-based cerebral vasospasm surveillance, Neurol. Res. Int. 2013, 256713.

Michel M., Jacob S., Roger G., Pelosse B., Laurier D., Ducou Le Pointe H., Bernier M.O. (2012) Eye lens radiation exposure and repeated head CT scans: A problem to keep in mind, Eur. J. Radiol. 81 (8), 1896-1900.

Société Française de Radiologie (2009) Guide pratique à l'usage des médecins radiologues pour l'évaluation de leurs pratiques professionnelles, ISBN : 978-2-916669-05-2.

Vajkoczy P., Horn P., Thome C., Munch E., Schmiedek P. (2003) Regional cerebral blood flow monitoring in the diagnosis of delayed ischemia following aneurysmal subarachnoid hemorrhage, Journal of Neurosurgery 98 (6), 1227-1234. 